\title{
On Synthesis of Orientation Insensitive Antennas
}

\author{
Kirill Klionovski, Maria Bermudez Arboleda, Zhen Su and Atif Shamim \\ Integrated Microwave Packaging Antennas and Circuits Technology Laboratory \\ King Abdullah University of Science and Technology (KAUST) \\ Thuwal, Kingdom of Saudi Arabia \\ kirill.klionovski@kaust.edu.sa, maria.bermudezarboleda@kaust.edu.sa, su.zhen@kaust.edu.sa, atif.shamim@kaust.edu.sa
}

\begin{abstract}
Internet of things applications require orientation insensitive wireless devices to maintain stable and reliable communication. The devices developed can also require positioning or a special protocol communication as Global Positioning System, Bluetooth Low Energy or Wi-Fi, which all implement a specific polarization, namely right hand circular (RHCP) and linear (LP) for the last two. For those reasons, antennas providing near-isotropic radiation patterns along with wide far-field sphere polarization coverage, including $L P$ and RHCP, are highly demanded in IoT applications. In this paper, we present some theoretical limitations on the synthesis of an antenna with near-isotropic radiation and polarization patterns. We consider two models: a set of orthogonal Hertzian electric dipoles located at different points, as well as a model of Hertzian magnetic dipoles located on faces of a perfectly electric conducting cube. These models allow synthesizing the near-isotropic radiation and polarization patterns of an antenna based on short dipoles, as well as a cubic antenna with radiating patches on the cube faces.
\end{abstract}

Keywords-orientation insensitive antenna; near-isotropic radiation pattern; near-isotropic polarization pattern.

\section{INTRODUCTION}

Internet of things (IoT) is a paradigm that becomes important in our daily life, where billions of stationary and moving electronic devices are connected to the Internet for smart applications. The significant number of devices and some newer applications demanding IoT capability on small items make special requirements to antennas: space efficiency, low cost, orientation insensitivity. The last condition is desirable to ensure that the device can function appropriately irrespective of its relative orientation to the base station, and can be accomplished by generating a near-isotropic radiation pattern of the antennas to avoid any loss due to nulls in the pattern. This should be implemented along with wide far-field sphere coverage by righthand circular polarization (RHCP) or linear polarization (LP), as these are necessary for receiving of Global Positioning System (GPS) or Bluetooth low energy (BLE) and Wi-Fi signals, respectively.

The near-isotropic radiation pattern is characterized by a parameter named isotropy, which is the variation of a total radiation pattern from the maximum to the minimum value. Meanwhile, the polarization pattern isotropy is calculated through the square of a far-field sphere that is covered by a specific type of polarization.
Some designs of antennas with near-isotropic radiation or polarization patterns have been previously published in $[1,2]$ or [3], respectively. However, synthesis of an antenna that provides both characteristics has not been considered in the literature.

An antenna that has near-isotropic radiation or polarization patterns, can be modeled based on electric or magnetic Hertzian dipoles distributions as investigated in [1-12]. For example, the assessment of concentric ring dipoles arrays was performed in [3-6]. A task of current synthesis on the surface of a sphere to obtain a near-isotropic radiation pattern was discussed in [7, 8]. The model of the sphere is applicable to a spherical antenna fed by an annular slot as shown in [9], which creates a radiation pattern with high isotropy. Some models of orthogonal electric dipoles were considered in literature to get quasi-isotropic radiation patterns: the model of a linear array of turnstile dipoles in [10], the model of orthogonal electric and magnetic dipoles in [11], and the model of a set of three orthogonal electric dipoles located at one point in $[1,12]$.

In this paper, we investigate a model of three orthogonal Hertzian electric dipoles located at different points and a model of Hertzian magnetic dipoles located on the faces of a perfectly electric conducting (PEC) cube. These models are used to synthesize the near-isotropic radiation and polarization patterns of a short dipoles antenna and a patches-on-cube antenna.

\section{THE LIMITATIONS ON RADIATION AND POLARIZATION PATTERNS ISOTROPY}

In the beginning, let us investigate the conditions of simultaneous realization of isotropic radiation and polarization patterns of an antenna. In [13], it was shown that an antenna creating an isotropic circular polarization $(\mathrm{CP})$ pattern has, at least, one null of a radiation pattern. If there are no nulls of a radiation pattern, then the antenna operates with linear polarization at least in one direction. The proof of this statement is based on the Brouwer theorem [14]. The theorem says that a one-valued vector, tangent to a simply connected surface and continuously changing on it, must be equal to zero or infinity at least at one point. Let us assume that an antenna has no nulls of radiation pattern and operates with $\mathrm{CP}$. In the far-field, the electric field intensity vector created by the antenna has meridional $E_{\theta}$ and azimuthal $E_{\varphi}$ components in the spherical coordinate system $(r, \theta, \varphi)$. These components are tangential to a sphere of radius $r$. From physical considerations, it is clear that $E_{\theta}$ is a one-valued vector, and it is continuous on the sphere. 
Besides, it can not be equal to infinity. After applying the Brouwer theorem to $E_{\theta}$, we obtain that $E_{\theta}=0$ at least at one point on the sphere. Thus, either $E_{\varphi}=0$ at this point and there is a null of the radiation pattern, or there is linear polarization at this point. By following the vectors continuity condition for the last case, we conclude that it is always possible to find a direction where the axial ratio is equal to any predetermined value. Therefore, due to the requirement of near-isotropy of a radiation pattern, we can not get $100 \%$ of the far-field sphere covered by a specific type of polarization. Note that any type of polarization is equivalent in terms of implementation possibilities. To prove the last statement, let us assume that we have an electric current distribution that creates a linearly polarized field everywhere. Then, by changing the electric current distribution to a magnetic one, we get a $90^{\circ}$-shifted linearly polarized field everywhere. A combination of these two distributions with certain amplitudes and phases gives us the field of a required type of polarization.

The Brower theorem was also used in $[15,16]$ to prove that the synthesis of a linearly polarized antenna with a null-free radiation pattern is impossible. However, in paper [17], it was shown that a two-port antenna excited by two independent sources could create fully isotropic radiation and polarization patterns simultaneously.

\section{THE SHORT DIPOLES ANTENNA}

The first proposed antenna consists of three short orthogonal dipoles as shown in Fig. 1(a). Each dipole is a strip of the length $L_{x}, L_{y}$, or $L_{z}$, which is fed in its center by a lumped port. The radiation pattern of the antenna can be calculated through a sum of radiation patterns of $\mathrm{X}-, \mathrm{Y}-$, and $\mathrm{Z}$-axis oriented electric Hertzian dipoles (Fig. 1(b)) with the unit magnitudes located at the points $P_{x}\left(\mathrm{X}_{x}, \mathrm{Y}_{x}, \mathrm{Z}_{x}\right), P_{y}\left(\mathrm{X}_{y}, \mathrm{Y}_{y}, \mathrm{Z}_{y}\right)$, and $P_{z}\left(\mathrm{X}_{z}, \mathrm{Y}_{z}, \mathrm{Z}_{z}\right)$, respectively. We assume that the $\mathrm{X}$ - and $\mathrm{Y}$-oriented dipoles have a phase shift $\psi$, and the $\mathrm{X}$ - and Z-oriented dipoles have a phase shift $\psi_{2}$. Then, the normalized meridional $E_{\theta}{ }^{\Sigma}$ and azimuthal $E_{\varphi}{ }^{\Sigma}$ components of the radiation pattern of the three Hertzian dipoles in the free space are calculated as follow:

$$
\begin{aligned}
& E_{\theta}^{\Sigma}(\theta, \varphi)=\cos \theta \cos \varphi \mathrm{e}^{i k r_{x}^{\prime} \cos \left(\alpha_{x}(\theta, \varphi)\right)}+ \\
& +\cos \theta \sin \varphi \mathrm{e}^{i k r_{y}^{\prime} \cos \left(\alpha_{y}(\theta, \varphi)\right)+i \psi}-\sin \theta \mathrm{e}^{i k r_{z}^{\prime} \cos \left(\alpha_{z}(\theta, \varphi)\right)+i \psi_{2}}, \\
& E_{\varphi}^{\Sigma}(\varphi)=-\sin \varphi \mathrm{e}^{i k r_{x}^{\prime} \cos \left(\alpha_{x}(\theta, \varphi)\right)}+\cos \varphi \mathrm{e}^{i k r_{y}^{\prime} \cos \left(\alpha_{y}(\theta, \varphi)\right)+i \psi} .
\end{aligned}
$$
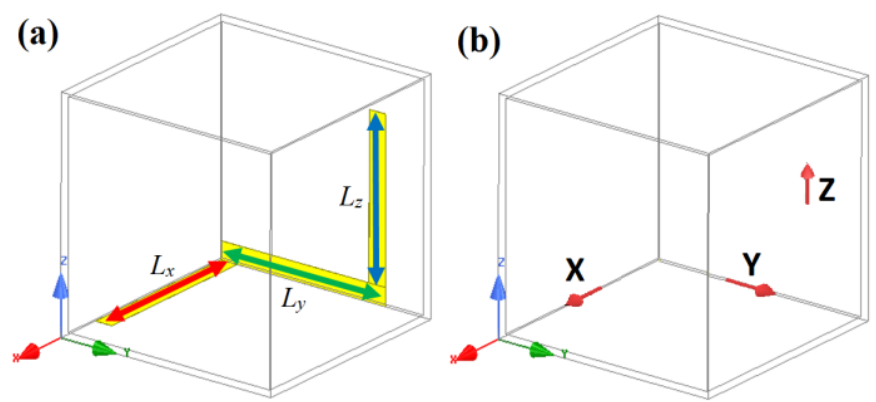

Fig. 1. (a) Short dipoles antenna; (b) Three Hertzian dipoles equivalent model.
Here, $k=2 \pi / \lambda, \lambda$ is free space wavelength, $i$ is the imaginary unit, and the location point of the $j$-oriented dipole is expressed through spherical coordinates as follow:

$$
\begin{aligned}
& r_{j}^{\prime}=\sqrt{X_{j}^{2}+Y_{j}^{2}+Z_{j}^{2}}, \quad \cos \left(\alpha_{j}(\theta, \varphi)\right)=\sin \theta \sin \theta_{j}^{\prime} \cos \left(\varphi-\varphi_{j}^{\prime}\right)+ \\
& +\cos \theta \cos \theta_{j}^{\prime}, \quad \theta_{j}^{\prime}=\arccos \left(\frac{Z_{j}}{r_{j}^{\prime}}\right), \quad \varphi_{j}^{\prime}=\arctan \left(\frac{Y_{j}}{X_{j}}\right) .
\end{aligned}
$$

Let us investigate the $\mathrm{CP}$ properties of the three Hertzian dipoles model. For this purpose, we introduce a normalized integral parameter based on an integration of the square of the far-field sphere that is covered by $\mathrm{CP}$ :

$I^{A R}=100 \% \times \int_{0}^{2 \pi} \int_{0}^{\pi} \frac{\sin \theta d \theta d \varphi}{4 \pi}$, if $\frac{1}{\sqrt{2}} \leq A R(\theta, \varphi) \leq 1$

The integration in (2) is performed only if the axial ratio (AR) in a specific direction is in the range of $1 / \sqrt{2} \leq A R \leq 1$. Expression of AR, which takes values in the range of $0 \leq A R \leq 1$, is given by [18]:

$$
\begin{aligned}
& A R=\sqrt{\frac{\left|E_{\theta}^{\Sigma}\right|^{2}+\left|E_{\varphi}^{\Sigma}\right|^{2}-E^{\sqrt{ }}}{\left|E_{\theta}^{\Sigma}\right|^{2}+\left|E_{\varphi}^{\Sigma}\right|^{2}+E^{\sqrt{ }}}}, \\
& E^{\sqrt{ }}=\sqrt{\left|E_{\theta}^{\Sigma}\right|^{4}+\left|E_{\varphi}^{\Sigma}\right|^{4}+2\left|E_{\theta}^{\Sigma}\right|^{2}\left|E_{\varphi}^{\Sigma}\right|^{2} \cos (2 \Delta \varphi)}, \\
& \Delta \varphi=\arg \left(E_{\theta}^{\Sigma}\right)-\arg \left(E_{\varphi}^{\Sigma}\right) .
\end{aligned}
$$

The RHCP and left-hand circular polarization (LHCP) corresponds to $\Delta \varphi>0$ and $\Delta \varphi<0$, respectively. We calculate the total radiation pattern as follow:

$\left|E^{\Sigma}(\theta, \varphi)\right|^{2}=\left|E_{\theta}^{\Sigma}(\theta, \varphi)\right|^{2}+\left|E_{\varphi}^{\Sigma}(\varphi)\right|^{2}$.

The phase shifts $\psi$ and $\psi_{2}$ were optimized to get maximum $I^{A R}$ for RHCP coverage along with isotropy of the dipoles located at the origin. The optimization results show that the maximum of $28.4 \%$ RHCP far-field sphere coverage is achieved for the values of $\psi=60^{\circ}$ and $\psi_{2}=300^{\circ}$. For such values of the phase shifts, the level of the cross LHCP coverage is only
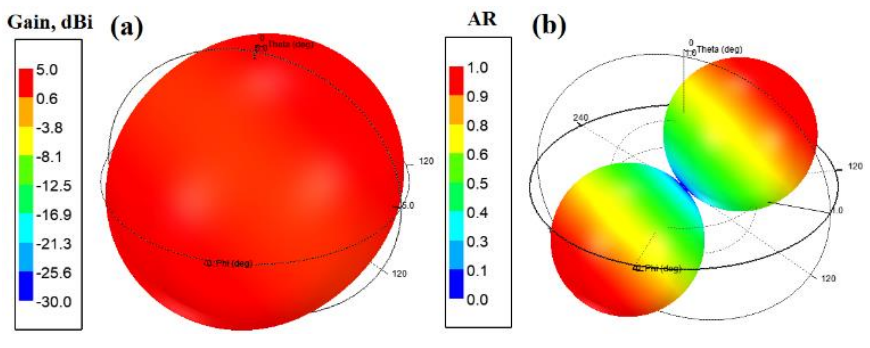

Fig. 2. (a) Radiation pattern of the three Hertzian dipoles; (b) AR pattern of the three Hertzian dipoles. 
$1 \%$, and the isotropy is $3 \mathrm{~dB}$. The model was simulated in Ansys HFSS software. Figure 2 shows the total radiation pattern and AR pattern for the optimized model of three dipoles located at the origin, and confirms the calculated values of $I^{A R}$ and isotropy.

\section{The PATCHES-ON-Cube ANTENNA}

The second design of an antenna with near-isotropic radiation and polarization patterns is presented in Fig. 3(a). The design
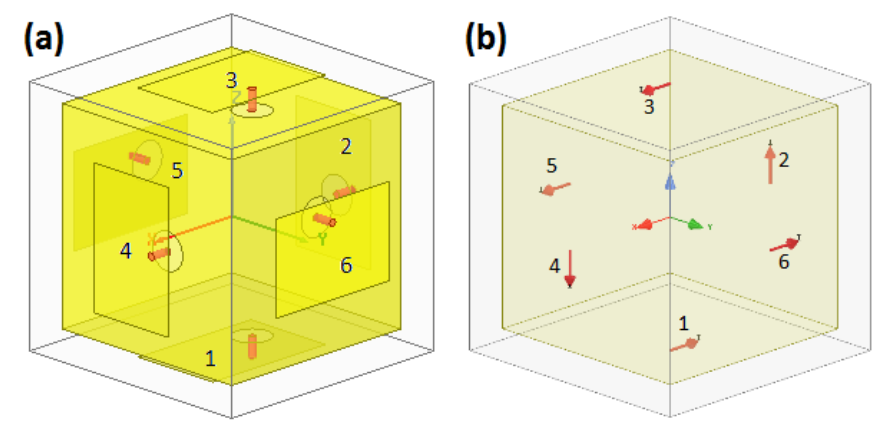

Fig. 3. (a) Patches-on-cube antenna; (b) Hertzian magnetic dipoles on the faces of the PEC cube.

consists of a PEC cube with the edge length of $\lambda / 2$. The radiating patches are placed on the faces of the cube. Each patch is fed by a separate feeding port with unit magnitude. The target is to synthesize near-isotropic radiation and LP patterns by changing the phase of each port excitation. To simplify the task of synthesis, let us model the radiation pattern of a patch by the radiation pattern of an equivalent current source. As it was shown in [19], the radiation pattern of a patch antenna placed on an infinite PEC ground plane coincides with the radiation pattern of an annular magnetic current above the ground plane. In the case when the size of the patch is very small, the annular current becomes a Hertzian magnetic dipole. Therefore, we represent each radiating patch through a Hertzian magnetic dipole (Fig. 3(b)) numbered from 1 to 6 . Thus, the task of synthesis is simplified to the determination of excitation phases of the dipoles. From the physical point of view, it is clear that we can obtain an omnidirectional radiation pattern in the plane of dipoles 1-4 location if we set the same phase excitation for each dipole 1-4. To get the near-isotropic radiation pattern in the perpendicular plane where dipoles 1, 5, 3, 6 are located, the phase of dipoles 5 and 6 should be $90^{\circ}$ shifted relative to the phase of dipoles 1-4.

The patches-on-cube antenna and the magnetic dipoles model were simulated in Ansys HFSS software when the excitation phase for each patch and a corresponding dipole was set as $0^{\circ}$ or $90^{\circ}$ for port number $1-4$ or 5 and 6 , respectively. The radiation and AR patterns are presented in Fig. 4. From this figure, we can see that the dipoles model results in appropriate predictions for the radiation and polarization patterns of the patches-on-cube antenna. The maximum and minimum gain of the antenna is 2.5 and $-3.6 \mathrm{dBi}$, respectively, yielding an
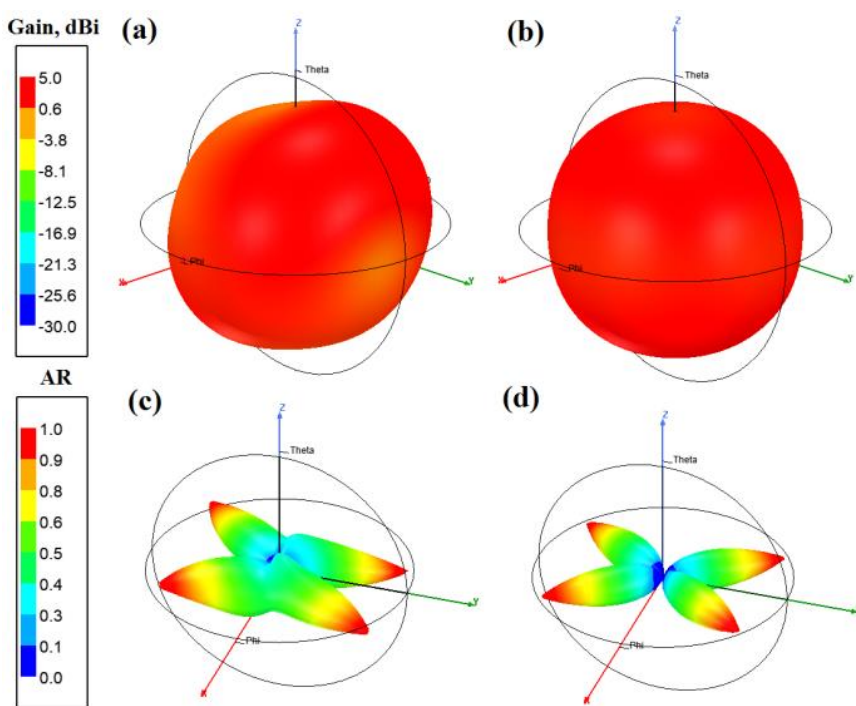

(d)

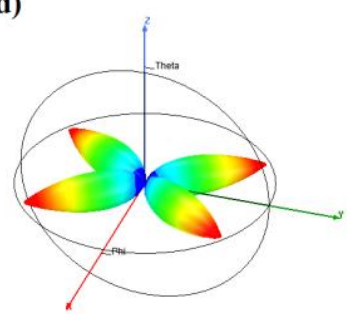

Fig. 4. (a) Radiation pattern of the patches-on-cube antenna; (b) Radiation pattern of the magnetic dipoles on cube; (c) AR pattern of the patches-on-cube antenna; (d) AR pattern of the magnetic dipoles on cube.

isotropy is $6.1 \mathrm{~dB}$, while more than $90 \%$ of LP coverage is achieved on the far-field sphere.

\section{CONCLUSIONS}

Given the obvious similarities on the simulations obtained for both proposed designs and their respective equivalent models of three orthogonal Hertzian electric dipoles and Hertzian magnetic dipoles on the PEC cube faces shown in Fig. 2 and Fig.4, we conclude that the use of these models are valid for simplifying the structures as well as for facilitating the synthesis of radiators that present both radiation and polarization isotropy as the three short dipoles antenna and the patches-oncube antenna presented.

\section{REFERENCES}

[1] Z. Su, K. Klionovski, R. M. Bilal, and A. Shamim, "A Dual Band Additively Manufactured 3-D Antenna on Package With Near-Isotropic Radiation Pattern," IEEE Trans. Antennas Propag., vol. 66, no. 7, pp. 3295-3305, July 2018.

[2] Z. Su, K. Klionovski, R. M. Bilal, and A. Shamim, "3D Printed Nearisotropic Asymmetric Dipole Antenna-on-Package for IoT Applications," in Proc. IEEE Indian Conference on Antennas and Propagation (InCAP), Hyderabad, India, Dec. 2018.

[3] G. H. Brown and O. M. Woodward, "Circularly-polarized omnidirectional antenna," RCA Review, vol. 8, no. 2, pp. 259-269, June 1947.

[4] H. L. Knudsen, "Radiation from ring quasi-arrays," IRE Trans. Antennas Propag., vol. 4, no. 3, pp. 452-472, July 1956.

[5] S. H. R. Zaidi, "On synthesis of isotropic patterns with concentric ring circular array," Tennessee Univ., Knoxwille, Engineering Experimental Station, Rept. 4 (AD-293868), Oct. 1962.

[6] O. M. Woodward, "A Mode Analysis of Quasi-Isotropic Antennas," RCA Review, vol. 26, no. 1, pp. 42-74, March 1965.

[7] A. Z. Fradin, "Synthesis of Radiators Most Closely Approximating an Isotropic System," Radio Engineering and Electronic Physics, vol. 8, no. 5, pp. 779-784, May 1963. 
[8] G. A. Postnov, "Synthesis of Antennas with Near-Isotropic Radiation Patterns," Radio Engineering and Electronic Physics, vol. 13, no. 2, pp. 190-194, Feb. 1968.

[9] D. S. Bugnolo, "A quasi-isotropic antenna in the microwave spectrum," IRE Trans. Antennas Propag., vol. 10, no. 4, pp. 377-383, Jul 1962.

[10] W. K. Saunders, "On the unity gain antenna," in Electromagnetic theory and antennas. Part 2. (ed. E. C. Jordan), pp. 1125-1130, New York, NY, USA: Pergamon press, 1963.

[11] Y. M. Pan, K. W. Leung, and K. Lu, "Compact quasi-isotropic dielectric resonator antenna with small ground plane," IEEE Trans. Antennas Propag., vol. 62, no. 2, pp. 577-585, Feb. 2014.

[12] F. F. Fulton, "The combined radiation pattern of three orthogonal dipoles", IEEE Trans. Antennas Propag, vol. 13, no. 2, pp. 323-324, March 1965.

[13] W. Scott and K. S. Hoo, "A theorem on the polarization of null-free antennas," IEEE Trans. Antennas Propag., vol. 14, no. 5, pp. 587-590, Sept. 1966.
[14] L. E. J. Brouwer, "On Continuous Vector Distributions on Surfaces," in Proc. of the Royal Netherlands Academy of Arts and Sciences (KNAW), Amsterdam, the Netherlands, vol. 11, pp. 850-858, 1909.

[15] H. F. Mathis, "A short proof that an isotropic antenna is impossible", Proc. IRE, vol. 39, p. 970, Aug. 1951.

[16] H. F. Mathis, "On isotropic antennas", Proc. IRE, vol. 42, p. 1810, Dec. 1954.

[17] G. A. Deschamps, J. D. Dyson, and P. E. Mast, "Two-Port Isotropic Radiator for Unpolarized Waves," IEEE Trans. Antennas Propag., vol. 17, no. 6, pp. 809-810, Nov. 1969.

[18] C. Balanis, Antenna Theory: Analysis and Design. The 4th Edition. Hoboken, NJ, USA: Wiley, 2016.

[19] D. R. Jackson, "Microstrip antennas," in Antenna engineering handbook. The 4th Edition (ed. J. Volakis). New York, NY, USA: McGraw Hill Companies. 2007. 